Supplementary Information

\title{
Factors controlling the risks of co-occurrence of the redox-sensitive elements of arsenic, chromium, vanadium, and uranium in groundwater from the eastern United States
}

Rachel M. Coyte†, Avner Vengosh†*

$\uparrow$ Nicholas School of the Environment, Duke University, Durham, North Carolina 27708, United States

*E-mail: vengosh@duke.edu. Phone: 919-681-8050. Fax: 919-684-5833.

\section{$\begin{array}{ll}\text { Contents } & \text { Page }\end{array}$}

1. Background on Duke Forest and Orange County Monitoring Wells........ S2

2. Tables (Table S1 to Table S7) .................................. S3

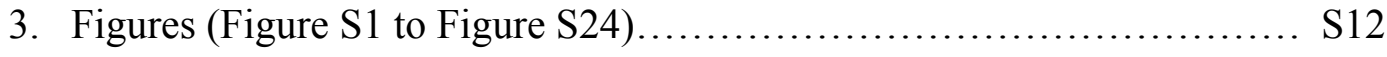

4. References................................................ S27 


\section{Background on Duke Forest and Orange County Monitoring Wells}

The monitoring wells from Duke Forest and Orange County investigated in this study lie within the Carolina Slate belt, but represent different formations. The Duke Forest wells are comprised of 4 well nests of 3 to 5 wells each, drilled at varying depths. At least one well in each nest was drilled in the saprolite, one in the transition zone between saprolite and bedrock, and one in the fractured bedrock. All well screens are 10ft long. Bedrock at nest DF-1 is medium grained diorite, at nest DF-2 immature volcanic sandstone to andesite/ basalt with depth, and granodiorite to quartz monzodiorite at nests DF-3 and DF-4 (Table S3). Wells from nest DF-3 are artesian. Among the 9 Orange County monitoring wells, there are two sets of paired wells (one saprolite, one bedrock), with the remaining five being single wells. Dacite, gabbro, mafic lavas and tuffs, altered tuffs, and epiclastics are represented by these wells (Table S3). A subset of the monitoring wells were measured in both the summer and the winter to assess possible seasonal differences. Though groundwater tables in North Carolina are generally lower in the summer, there was no significant difference between either water levels or RSE concentrations between the two sampling events (Figures S14 and S15). 
Table S1. Description of major geological belts in North Carolina

\begin{tabular}{|c|c|}
\hline Belt & Description \\
\hline Kings Mountain Belt & $\begin{array}{l}\text { Moderately metamorphosed volcanic and } \\
\text { sedimentary rock }\end{array}$ \\
\hline Charlotte and Milton Belts & $\begin{array}{l}\text { Intrusives and meta-igneous rocks. More southern } \\
\text { Charlotte belt is mostly granite, diorite, and gabbro, } \\
\text { and Milton belt to the North is more gneiss, schist, } \\
\text { and metamorphosed material. }\end{array}$ \\
\hline Triassic Basins & $\begin{array}{c}\text { Sedimentary rocks, mostly mudstones, some intruded } \\
\text { igneous material. }\end{array}$ \\
\hline Raleigh Belt & $\begin{array}{c}\text { Meta-igneous rocks, mostly, granite, gneiss, and } \\
\text { schist }\end{array}$ \\
\hline $\begin{array}{l}\text { Inner Piedmont, Chauga Belt, Smith River } \\
\text { Allochthon, and Sauratown Mountain }\end{array}$ & $\begin{array}{l}\text { Highly metamorphosed sedimentary and igneous } \\
\text { rock with some younger granite intrusions }\end{array}$ \\
\hline Carolina Slate Belt & Metamorphosed sedimentary and volcanic rock \\
\hline Blue Ridge Belt & $\begin{array}{l}\text { Complex mixture of igneous, sedimentary, and } \\
\text { metamorphic rock }\end{array}$ \\
\hline Coastal Plain & $\begin{array}{l}\text { Primarily marine sedimentary rocks, especially sand } \\
\text { and clay, thickening to the east }\end{array}$ \\
\hline Eastern Slate Belt & $\begin{array}{c}\text { Slightly metamorphosed volcanic and sedimentary } \\
\text { rock }\end{array}$ \\
\hline
\end{tabular}


Table S2: Sources of data used in this manuscript. Numbers represent the number of observations used from each data source. Due to the multiple detection limits in the compiled dataset, all total As, Cr, U, V data was given an artificial detection limit of $0.5 \mu \mathrm{g} / \mathrm{L}$ for the purposes of analysis. Samples for $\mathrm{Cr}(\mathrm{VI})$ were assigned a detection limit of $0.07 \mu \mathrm{g} / \mathrm{L}$. A few samples from the NC Dept. of Environmental Quality dataset were censored for having detection limits above these assigned limits.

\begin{tabular}{|l|l|l|l|l|l|}
\hline $\begin{array}{l}\text { Source/ } \\
\text { Number of } \\
\text { Observations }\end{array}$ & Arsenic & Chromium & Uranium & Vanadium & Chromium (VI) \\
\hline $\begin{array}{l}\text { Duke } \\
\text { University }\end{array}$ & 595 & 604 & 598 & 600 & 224 \\
\hline $\begin{array}{l}\text { NC Dept. of } \\
\text { Environmental } \\
\text { Quality }\end{array}$ & 322 & 335 & 0 & 261 & 327 \\
\hline $\begin{array}{l}\text { US } \\
\text { Environmental } \\
\text { Protection } \\
\text { Agency } \\
\text { UCMR3 }\end{array}$ & 0 & 317 & 0 & 132 & 317 \\
\hline $\begin{array}{l}\text { United State } \\
\text { Geological } \\
\text { Survey }\end{array}$ & 136 & 92 & 122 & 124 & 0 \\
\hline
\end{tabular}


Table S3. Duke Monitoring Well construction, geology, and chemical data.

\begin{tabular}{|c|c|c|c|c|c|c|c|c|c|c|c|c|c|}
\hline Name & Cluster & Bedrock & $\begin{array}{l}\text { Depth } \\
\text { Category }\end{array}$ & $\begin{array}{l}\text { Water } \\
\text { Level } \\
(\mathbf{m})\end{array}$ & $\begin{array}{l}\text { Depth } \\
\text { to Top } \\
\text { of } \\
\text { Screen } \\
\text { (m) }\end{array}$ & Season & pH & $\begin{array}{l}\text { DO } \\
(\mathrm{mg} / \mathrm{L})\end{array}$ & $\begin{array}{l}\mathbf{U} \\
\mu \mathrm{g} / \mathrm{L}\end{array}$ & $\begin{array}{l}\text { As } \\
\mu \mathrm{g} / \mathrm{L}\end{array}$ & $V \mu \mathrm{g} / \mathrm{L}$ & $\mathrm{Cr} \mu \mathrm{g} / \mathrm{L}$ & $\begin{array}{l}\operatorname{Cr}(V I) \\
\mu g / L\end{array}$ \\
\hline $911 C$ & $911 \mathrm{C}$ & Altered Tuff & Deep & 21 & 85 & Winter & 6.14 & 0.65 & Bdl & 0.75 & 0.04 & Bdl & Bdl \\
\hline BF-D & $\mathrm{BF}$ & $\begin{array}{l}\text { Felsic Lavas } \\
\text { and Tuffs } \\
\text { (Dacite) }\end{array}$ & Deep & 21.2 & 100 & Winter & 6.85 & 0.39 & 0.14 & 0.13 & 0.03 & Bdl & Bdl \\
\hline BF-S & $\mathrm{BF}$ & $\begin{array}{l}\text { Felsic Lavas } \\
\text { and Tuffs } \\
\text { (Dacite) }\end{array}$ & Saprolite & 18 & 30 & Winter & 6.8 & 6.06 & Bdl & Bdl & 0.03 & Bdl & 0.22 \\
\hline BRUM & BRUM & $\begin{array}{l}\text { Mafic Lavas } \\
\text { and Tuffs }\end{array}$ & Deep & 28.3 & 108 & Winter & 7.52 & 0.15 & $\mathrm{Bdl}$ & 2.61 & 0.02 & Bdl & Bdl \\
\hline DF-1D & DF-1 & Diorite & Deep & 59.4 & 62 & Summer & 6.37 & 4.63 & Bdl & 0.07 & 2.31 & 0.43 & 0.33 \\
\hline DF-1D & DF-1 & Diorite & Deep & 57.3 & 62 & Winter & 6.66 & 4.07 & Bdl & 0.14 & 2.65 & 0.35 & 0.46 \\
\hline DF-1I & DF-1 & Diorite & Transition & 41.4 & 42 & Winter & 5.98 & 6.3 & Bdl & 0.04 & 1.97 & 0.36 & 0.53 \\
\hline $\begin{array}{l}\text { DF- } \\
2 \mathrm{CH}\end{array}$ & DF-2 & Mafic Tuffs & Transition & 58 & 30 & Summer & 5.66 & 4.43 & 0.04 & 0.17 & 1.33 & 3.73 & 3.72 \\
\hline $\begin{array}{l}\text { DF- } \\
\text { 2DP }\end{array}$ & DF-2 & Mafic Tuffs & Deep & 57.4 & 62 & Summer & 7.29 & 0.43 & 0.03 & 1.45 & 0.04 & Bdl & 0.03 \\
\hline $\begin{array}{l}\text { DF- } \\
\text { 2DS }\end{array}$ & DF-2 & Mafic Tuffs & Deep & 56 & 90 & Summer & 7.19 & 0.46 & 0.02 & 1.81 & 0.07 & Bdl & 0.01 \\
\hline $\begin{array}{l}\text { DF- } \\
\text { 2DS }\end{array}$ & DF-2 & Mafic Tuffs & Deep & 56.8 & 87 & Winter & 7.66 & 0.62 & Bdl & 2.54 & 0.06 & Bdl & Bdl \\
\hline DF-2I & DF-2 & Mafic Tuffs & Transition & 53.4 & 50 & Winter & 5.81 & 5.25 & Bdl & Bdl & 0.69 & Bdl & 0.11 \\
\hline $\begin{array}{l}\text { DF- } \\
\text { 3CH }\end{array}$ & DF-3 & Granodiorite & Saprolite & artesian & 9 & Summer & 7 & 0.59 & 0.29 & 0.17 & 0.24 & Bdl & Bdl \\
\hline $\begin{array}{l}\text { DF- } \\
\text { 3CH }\end{array}$ & DF-3 & Granodiorite & Saprolite & artesian & 9 & Winter & 7.22 & 0.24 & 0.24 & Bdl & 0.13 & Bdl & 0.01 \\
\hline DF-3D & DF-3 & Granodiorite & Deep & artesian & 86 & Summer & 7.17 & 0.8 & 0.57 & 0.19 & 0.08 & Bdl & Bdl \\
\hline DF-3D & DF-3 & Granodiorite & Deep & artesian & 83 & Winter & 7.34 & 0.2 & 0.55 & 0.10 & Bdl & Bdl & Bdl \\
\hline DF-3I & DF-3 & Granodiorite & Transition & artesian & 35 & Summer & 7.06 & 0.56 & 0.55 & 0.14 & 0.12 & Bdl & 0.08 \\
\hline DF-3I & DF-3 & Granodiorite & Transition & artesian & 35 & Winter & 7.35 & 0.18 & 0.52 & 0.07 & 0.13 & Bdl & $\mathrm{Bdl}$ \\
\hline DF-3S & DF-3 & Granodiorite & Saprolite & artesian & 10 & Summer & 7.25 & 0.54 & 0.47 & 0.33 & 0.63 & Bdl & Bdl \\
\hline DF-3S & DF-3 & Granodiorite & Saprolite & artesian & 10 & Winter & 7.21 & 1.94 & 0.47 & 0.30 & 0.62 & Bdl & Bdl \\
\hline
\end{tabular}




\begin{tabular}{|c|c|c|c|c|c|c|c|c|c|c|c|c|c|}
\hline DF-4D & DF-4 & $\begin{array}{l}\text { Granodiorite/ } \\
\text { Quartz } \\
\text { Monzodiorite }\end{array}$ & Deep & 19.9 & 92 & Summer & 7.07 & 0.35 & 0.22 & 0.20 & 0.27 & $\mathrm{Bdl}$ & 0.11 \\
\hline DF-4D & DF-4 & $\begin{array}{l}\text { Granodiorite/ } \\
\text { Quartz } \\
\text { Monzodiorite }\end{array}$ & Deep & 20.8 & 85 & Winter & 7.34 & 0.46 & 0.16 & 0.21 & 0.36 & 0.29 & $\mathrm{Bdl}$ \\
\hline DF-4I & DF-4 & $\begin{array}{l}\text { Granodiorite/ } \\
\text { Quartz } \\
\text { Monzodiorite }\end{array}$ & Transition & 22.1 & 26 & Summer & 5.44 & 0.58 & 0.04 & 0.12 & 0.50 & 0.31 & $\mathrm{Bdl}$ \\
\hline DF-4I & DF-4 & $\begin{array}{l}\text { Granodiorite/ } \\
\text { Quartz } \\
\text { Monzodiorite }\end{array}$ & Transition & 21.8 & 26 & Winter & 5.56 & 0.45 & Bdl & 0.14 & 0.24 & Bdl & $\mathrm{Bdl}$ \\
\hline ENO & ENO & Felsic Tuff & Transition & 30 & 37 & Winter & 6.8 & 0.32 & 0.01 & Bdl & 0.07 & Bdl & Bdl \\
\hline EUB & EUB & $\begin{array}{l}\text { Mafic } \\
\text { Plutonics } \\
\text { (Gabbro) }\end{array}$ & Transition & 8.5 & 33 & Winter & 7.02 & 0.32 & Bdl & 0.28 & 0.05 & 0.278347 & $\mathrm{Bdl}$ \\
\hline MILL & MILL & Epiclastics & Deep & 27 & 67 & Winter & 6.37 & 0.22 & Bdl & 0.78 & 0.07 & Bdl & Bdl \\
\hline NED & NEP & Epiclastics & Deep & 28.3 & 43.5 & Winter & 7.14 & 2.08 & Bdl & 0.80 & 0.60 & Bdl & 0.05 \\
\hline NES-S & NEP & Epiclastics & Saprolite & 18 & 12 & Winter & 5.67 & 6.97 & $\mathrm{Bdl}$ & Bdl & 0.11 & Bdl & 0.23 \\
\hline
\end{tabular}


Table S4. Probability of exceeding $0.5 \mu \mathrm{g} / \mathrm{L}$ for $\mathrm{As}, \mathrm{Cr}, \mathrm{U}$, and $\mathrm{V}$ for geologic belts.

\begin{tabular}{|l|l|l|l|l|}
\hline Belt & Arsenic (n) & Chromium (n) & Uranium (n) & Vanadium (n) \\
\hline Charlotte and Milton Belts & $0.23(461)$ & $0.68(471)$ & $0.26(209)$ & $0.87(433)$ \\
\hline Carolina Slate Belt & $0.54(192)$ & $0.48(186)$ & $0.24(187)$ & $0.64(189)$ \\
\hline Raleigh Belt & $0.10(123)$ & $0.42(212)$ & $0.39(124)$ & $0.71(184)$ \\
\hline Triassic Basins & $0.6(29)$ & $0.38(25)$ & $0.38(28)$ & $0.84(18)$ \\
\hline Coastal Plain & $0.14(154)$ & $0.13(309)$ & $0.05(127)$ & $0.30(199)$ \\
\hline Blue Ridge Belt & $0(25)$ & $0.09(33)$ & $0.04(24)$ & $0.43(22)$ \\
\hline Inner Piedmont and Related Belts & $0.16(30)$ & $0.44(71)$ & $0.26(18)$ & $0.52(41)$ \\
\hline Eastern Slate Belt & $0.14(6)$ & $0.11(8)$ & $0.0(2)$ & $0.67(5)$ \\
\hline Kings Mountain Belt & $0(32)$ & $0.76(33)$ & $0.0(0)$ & $0.96(25)$ \\
\hline Grandfather Mountain Window & $0(1)$ & $0.0(0)$ & $0.0(1)$ & $0.0(1)$ \\
\hline
\end{tabular}


Table S5. Exceedance probabilities of all possible contaminant combinations, sorted by belt. Number of samples in each category shown in parenthesis.

\begin{tabular}{|c|c|c|c|c|c|c|c|c|c|c|c|}
\hline Belt & As-Cr(VI) & As-U & As-V & $\begin{array}{l}\mathrm{Cr}(\mathrm{VI})- \\
\mathrm{V}\end{array}$ & $\begin{array}{l}\mathrm{Cr}(\mathrm{VI})- \\
\mathrm{U}\end{array}$ & $\mathbf{U}-\mathbf{V}$ & $\begin{array}{l}\text { As-Cr(VI)- } \\
\text { V }\end{array}$ & $\begin{array}{l}\text { As-U- } \\
\text { Cr(VI) }\end{array}$ & As-U-V & $\begin{array}{l}\text { U- } \\
\operatorname{Cr}(V I)- \\
\text { V }\end{array}$ & $\begin{array}{l}\text { As-V- } \\
\text { U- } \\
\operatorname{Cr}(V I)\end{array}$ \\
\hline Blue Ridge Belt & $0(23)$ & $0(17)$ & $\begin{array}{l}0 \\
(16)\end{array}$ & $0.35(19)$ & $0(16)$ & $0.06(17)$ & $0(16)$ & $0(16)$ & $0(16)$ & $0(16)$ & $0(16)$ \\
\hline Carolina Slate Belt & $0.04(80)$ & $0.21(182)$ & $\begin{array}{l}0.44 \\
(180)\end{array}$ & $0.35(84)$ & $0.02(80)$ & $\begin{array}{l}0.23 \\
(179)\end{array}$ & $0.04(80)$ & $0.02(80)$ & $0.21(174)$ & $0.02(80)$ & $\begin{array}{l}0.02 \\
(80)\end{array}$ \\
\hline $\begin{array}{l}\text { Charlotte and } \\
\text { Milton Belts }\end{array}$ & $0.06(323)$ & $0.15(204)$ & $\begin{array}{l}0.14 \\
(413) \\
\end{array}$ & $\begin{array}{l}0.84 \\
(297)\end{array}$ & $0.12(82)$ & $\begin{array}{l}0.19 \\
(206)\end{array}$ & $0.06(286)$ & $0.04(82)$ & $0.11(201)$ & $0.21(82)$ & $\begin{array}{l}0.04 \\
(82) \\
\end{array}$ \\
\hline Coastal Plain & $0(22)$ & $0.02(116)$ & $\begin{array}{l}0.11 \\
(134) \\
\end{array}$ & $0.27(44)$ & $0(9)$ & $\begin{array}{l}0.06 \\
(122)\end{array}$ & $0(11)$ & $0(9)$ & $0.02(115)$ & $0(9)$ & $0(9)$ \\
\hline Eastern Slate Belt & $0(5)$ & $0(2)$ & $0(3)$ & $0.4(4)$ & $0(1)$ & $0(2)$ & $0(2)$ & $0(1)$ & $0(2)$ & $0(1)$ & $0(1)$ \\
\hline $\begin{array}{l}\text { Grandfather } \\
\text { Mountain Window }\end{array}$ & $0(0)$ & $0 \quad(1)$ & $0(1)$ & $0(0)$ & $0(0)$ & $0(1)$ & $0(0)$ & $0(0)$ & $0(1)$ & $0(0)$ & $0(0)$ \\
\hline $\begin{array}{l}\text { Inner Piedmont and } \\
\text { Related Belts }\end{array}$ & $0(19)$ & $0(10)$ & $0(9)$ & $0.47(33)$ & $0.13(7)$ & $0.14(13)$ & $0(8)$ & $0(7)$ & $0(8)$ & $0.13(7)$ & $0(7)$ \\
\hline $\begin{array}{l}\text { Kings Mountain } \\
\text { Belt }\end{array}$ & $0(32)$ & $0(0)$ & $\begin{array}{l}0 \\
(24)\end{array}$ & $0.84(24)$ & $0(0)$ & $0(0)$ & $0(24)$ & $0(0)$ & $0(00$ & $0(0)$ & $0(0)$ \\
\hline Raleigh Belt & $0(14)$ & $0.02(116)$ & $\begin{array}{l}0.07 \\
(116) \\
\end{array}$ & $0.73(79)$ & $0.33(14)$ & $\begin{array}{l}0.36 \\
(119) \\
\end{array}$ & $0(14)$ & $0(14)$ & $0.02(116)$ & $0.33(14)$ & $0(14)$ \\
\hline Triassic Basins & $0.35(16)$ & $0.31(28)$ & $\begin{array}{l}0.42 \\
(18)\end{array}$ & $0.63(15)$ & $0.25(15)$ & $0.32(18)$ & $0.38(15)$ & $0.25(15)$ & $0.21(18)$ & $0.25(15)$ & $0.25(15)$ \\
\hline
\end{tabular}


Table S6. Exceedance probabilities of health guidelines, divided by belt, with number of observations in parenthesis.

\begin{tabular}{|l|r|r|r|r|}
\hline & $\begin{array}{l}\text { Exceedance } \\
\text { probability of 1 } \\
\text { or more } \\
\text { contaminants }\end{array}$ & $\begin{array}{l}\text { Exceedance } \\
\text { probability of } \\
\text { 2 or more } \\
\text { contaminants }\end{array}$ & $\begin{array}{l}\text { Exceedance } \\
\text { probability of } \\
\text { 3 or more } \\
\text { contaminants }\end{array}$ & $\begin{array}{l}\text { Exceedance } \\
\text { probability of 4 } \\
\text { contaminants }\end{array}$ \\
\hline Blue Ridge Belt & $0.37(40)$ & $0.28(28)$ & $0.00(16)$ & $0.00(16)$ \\
\hline Carolina Slate Belt & $0.87(207)$ & $0.56(0.56)$ & $0.22(174)$ & $0.02(80)$ \\
\hline $\begin{array}{l}\text { Charlotte and Milton } \\
\text { Belts }\end{array}$ & $0.93(488)$ & $0.68(469)$ & $0.10(405)$ & $0.04(82)$ \\
\hline Coastal Plain & $0.36(378)$ & $0.17(186)$ & $0.02(117)$ & $0.00(9)$ \\
\hline Eastern Slate Belt & $0.56(8)$ & $0.22(8)$ & $0.00(3)$ & $0.00(1)$ \\
\hline $\begin{array}{l}\text { Grandfather } \\
\text { Mountain Window }\end{array}$ & $0.50(1)$ & $0.00(1)$ & $0.00(1)$ & $0.00(0)$ \\
\hline $\begin{array}{l}\text { Inner Piedmont and } \\
\text { Related Belts }\end{array}$ & $0.54(83)$ & $0.32(52)$ & $0.10(9)$ & $0.00(7)$ \\
\hline Kings Mountain Belt & $0.84(36)$ & $0.64(32)$ & $0.00(24)$ & $0.00(0)$ \\
\hline Raleigh Belt & $0.79(224)$ & $0.55(184)$ & $0.06(116)$ & $0.00(14)$ \\
\hline Triassic Basins & $0.9(29)$ & $0.63(29)$ & $0.32(18)$ & $0.25(15)$ \\
\hline
\end{tabular}


Table S7. Exceedance probabilities of enforceable regulatory standards, divided by belt, with number of observations in parenthesis.

\begin{tabular}{|l|l|l|l|}
\hline & $\begin{array}{l}\text { Exceedance } \\
\text { probability of } \\
1 \text { or more } \\
\text { contaminants }\end{array}$ & $\begin{array}{l}\text { Exceedance } \\
\text { probability of } \\
\text { 2 or more } \\
\text { contaminants }\end{array}$ & $\begin{array}{l}\text { Exceedance } \\
\text { probability of } \\
\text { or more } \\
\text { contaminants }\end{array}$ \\
\hline Blue Ridge Belt & $0.0(40)$ & $0.0(38)$ & $0.0(27)$ \\
\hline Carolina Slate Belt & $0.17(207)$ & $0.0(207)$ & $0.0(193)$ \\
\hline Charlotte and Milton Belts & $0.06(488)$ & $0.0(480)$ & $0.0(460)$ \\
\hline Coastal Plain & $0.002(378)$ & $0.0(351)$ & $0.0(139)$ \\
\hline Eastern Slate Belt & $0.0(8)$ & $0.0(8)$ & $0.0(6)$ \\
\hline Grandfather Mountain Window & $0.0(1)$ & $0.0(1)$ & $0.0(1)$ \\
\hline $\begin{array}{l}\text { Inner Piedmont and Related } \\
\text { Belts }\end{array}$ & $0.01(83)$ & $0.0(79)$ & $0.0(32)$ \\
\hline Kings Mountain Belt & $0.0(36)$ & $0.0(33)$ & $0.0(32)$ \\
\hline Raleigh Belt & $0.03(224)$ & $0.0(224)$ & $0.0(121)$ \\
\hline Triassic Basins & $0.03(29)$ & $0.0(29)$ & $0.0(29)$ \\
\hline
\end{tabular}

Table 8. Average values and standard deviations of reference material for the DCP-OES (cations) and IC (anions). Cations reps are diluted NIST $1643 F$, and anion reps are of Dionex 7 anion standard.

\begin{tabular}{|c|c|c|c|c|c|c|c|c|}
\hline & $\mathrm{Ca}(\mathrm{n}=48)$ & $M g(n=48)$ & $\mathrm{Na}(\mathrm{n}=48)$ & Fe $(n=48)$ & $M n(n=48)$ & $\mathrm{Cl}^{-}(\mathrm{n}=26)$ & $\mathrm{NO}_{3}{ }^{-}(\mathrm{n}=22)$ & $\mathrm{SO}_{4}{ }^{2-}(\mathrm{n}=26)$ \\
\hline $\begin{array}{l}\text { Certified } \\
\text { Value }\end{array}$ & 29.4 & 7.45 & 18.8 & 0.093 & 0.037 & 10 & 10 & 10 \\
\hline Average & 29.2 & 7.66 & 19.9 & 0.09 & 0.041 & 10.3 & 11.6 & 10.2 \\
\hline Std dev & 2.0 & 0.3 & 1.1 & 0.02 & 0.009 & 0.4 & 1.2 & 0.4 \\
\hline
\end{tabular}


Table 9. Results from Duke University's ICP-MS on a recent USGS inter-laboratory comparison.

\begin{tabular}{|l|c|c|c|c|}
\hline & $\operatorname{Cr}(n=10)$ & As $(n=10)$ & $V(n=10)$ & $U(n=10)$ \\
\hline $\begin{array}{l}\text { Most } \\
\text { Probable } \\
\text { Value }\end{array}$ & 3.06 & 5.36 & 4.08 & 1.68 \\
\hline Average & 3.06 & 5.30 & 4.12 & 1.77 \\
\hline Std dev & 0.06 & 0.09 & 0.07 & 0.03 \\
\hline
\end{tabular}




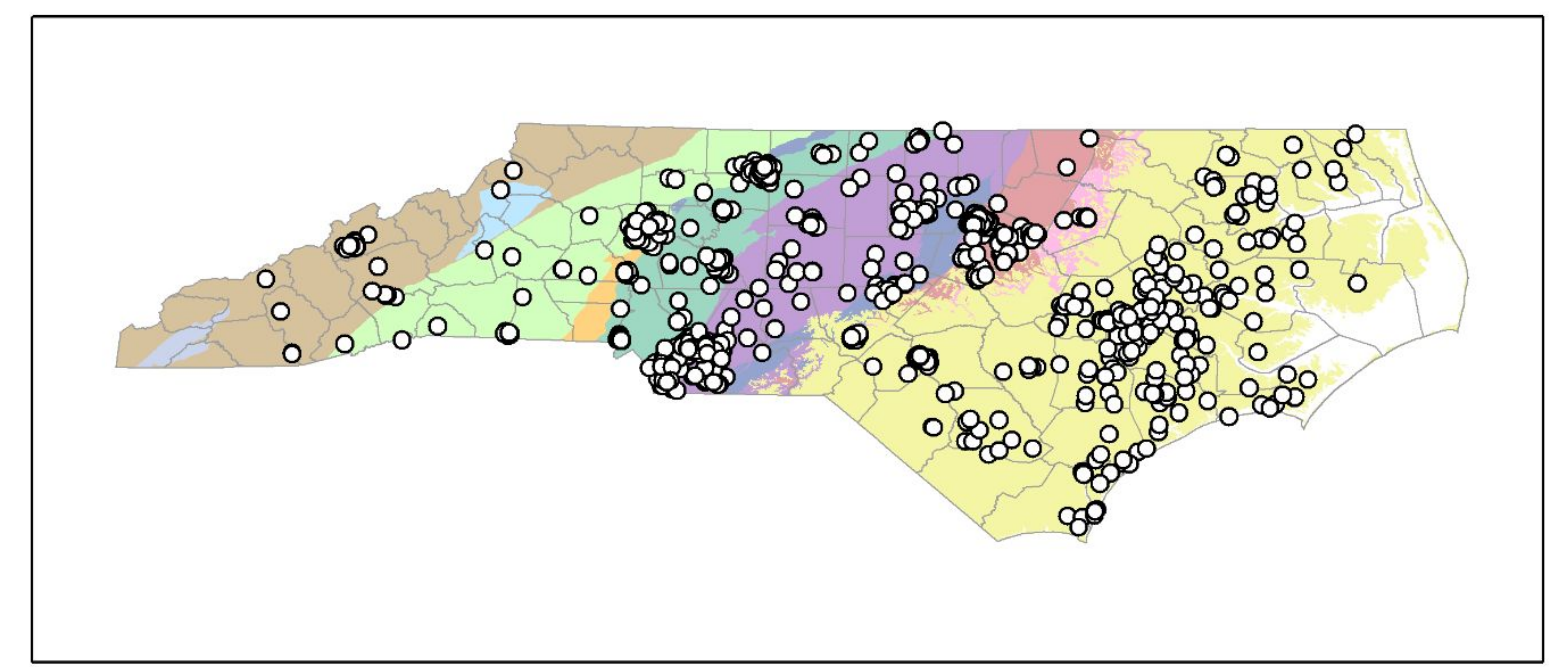

Belt

Blue Ridge Belt
Carolina Slate Belt

Coastal Plain

Eastern Slate Belt

Kings Mountain Belt

Grandfather Mountain Window

Murphy Belt

Charlotte and Milton Belts

Inner Piedmont, Chauga Belt, Smith River Allochthon, and Sauratown Mountain

Raleigh Belt

Figure S1. Sampling Locations overlaying North Carolina Belt Geology 


\section{Cations}

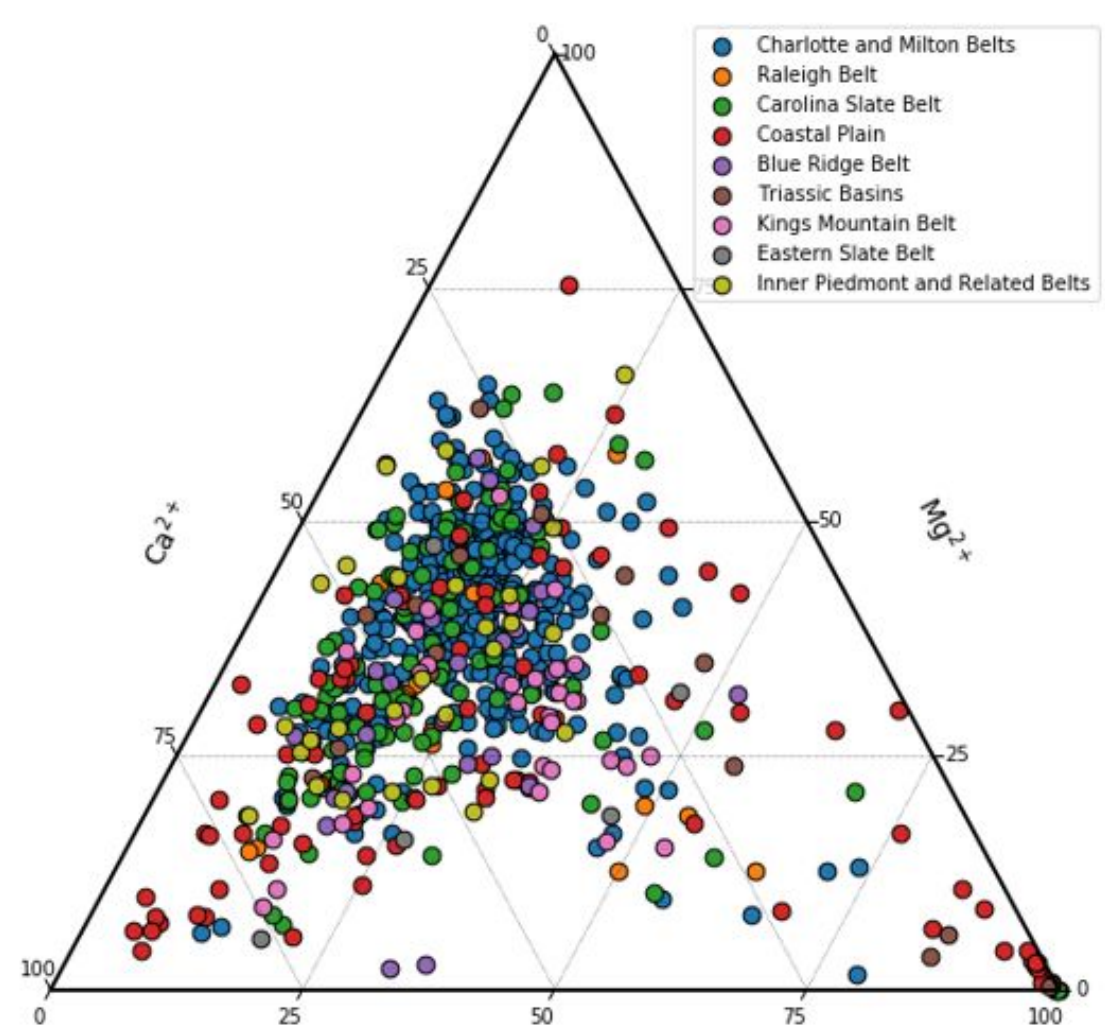

$\mathrm{Na}^{+}$
Anions

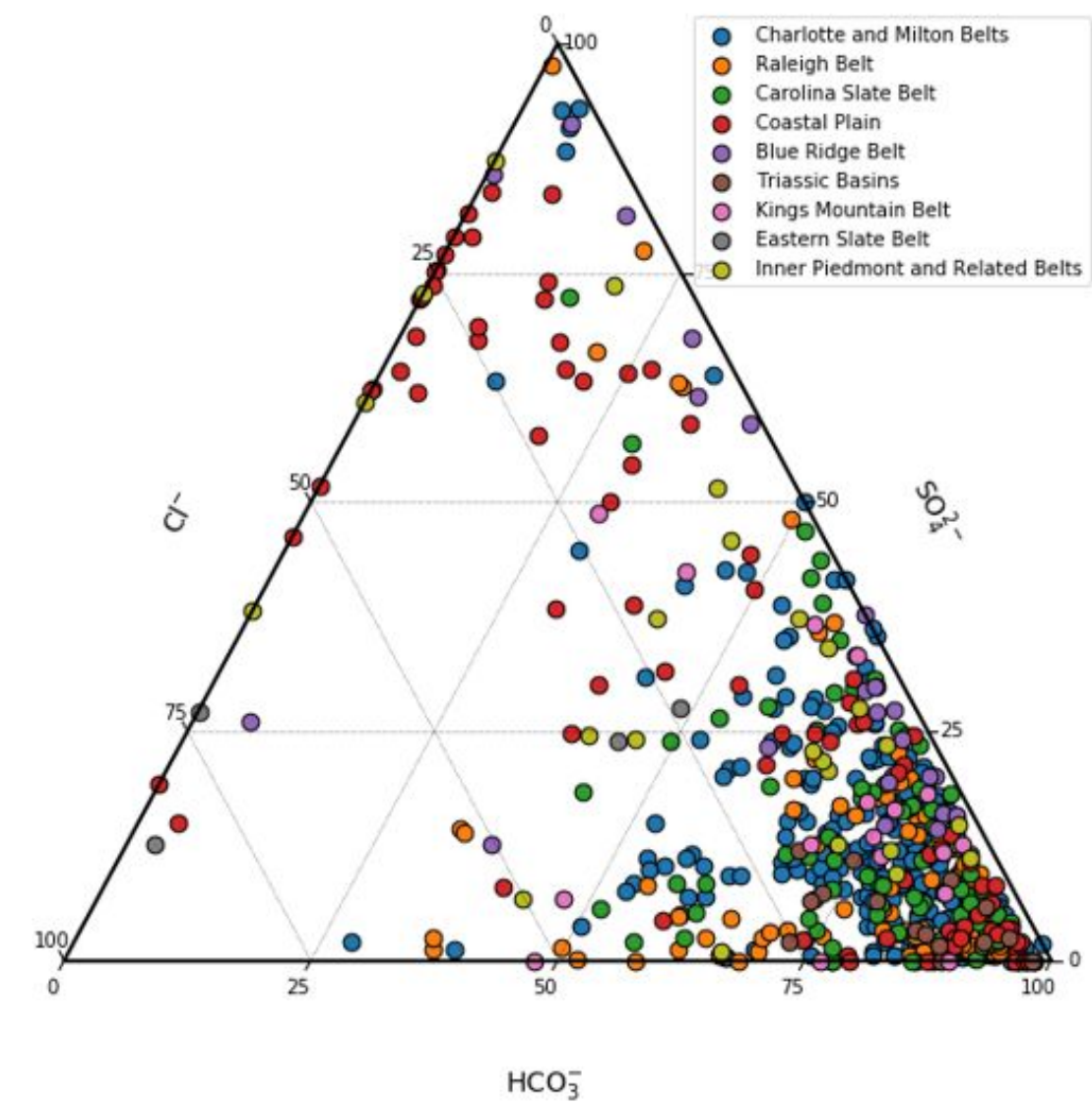

Figure 2. Ternary diagrams for North Carolina groundwater samples analyzed, separated by belt. 


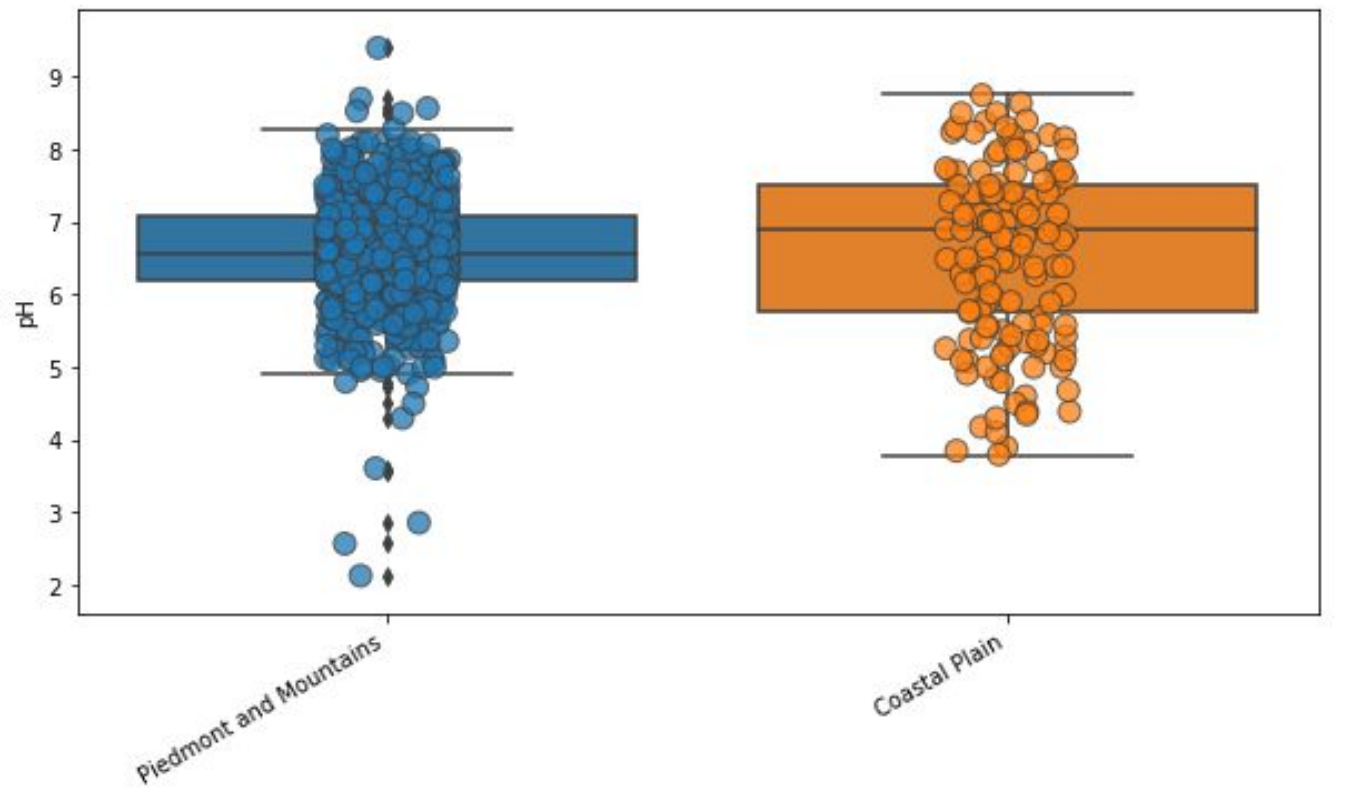

Figure S3. Boxplots of $\mathrm{pH}$ and Dissolved Oxygen (DO) concentrations for the Coastal Plain and Piedmont and Mountain Belts. Median DO Is higher in the Pidemont and mountain regions and median $p H$ is higher in the Coastal Plain (pval<0.01). 

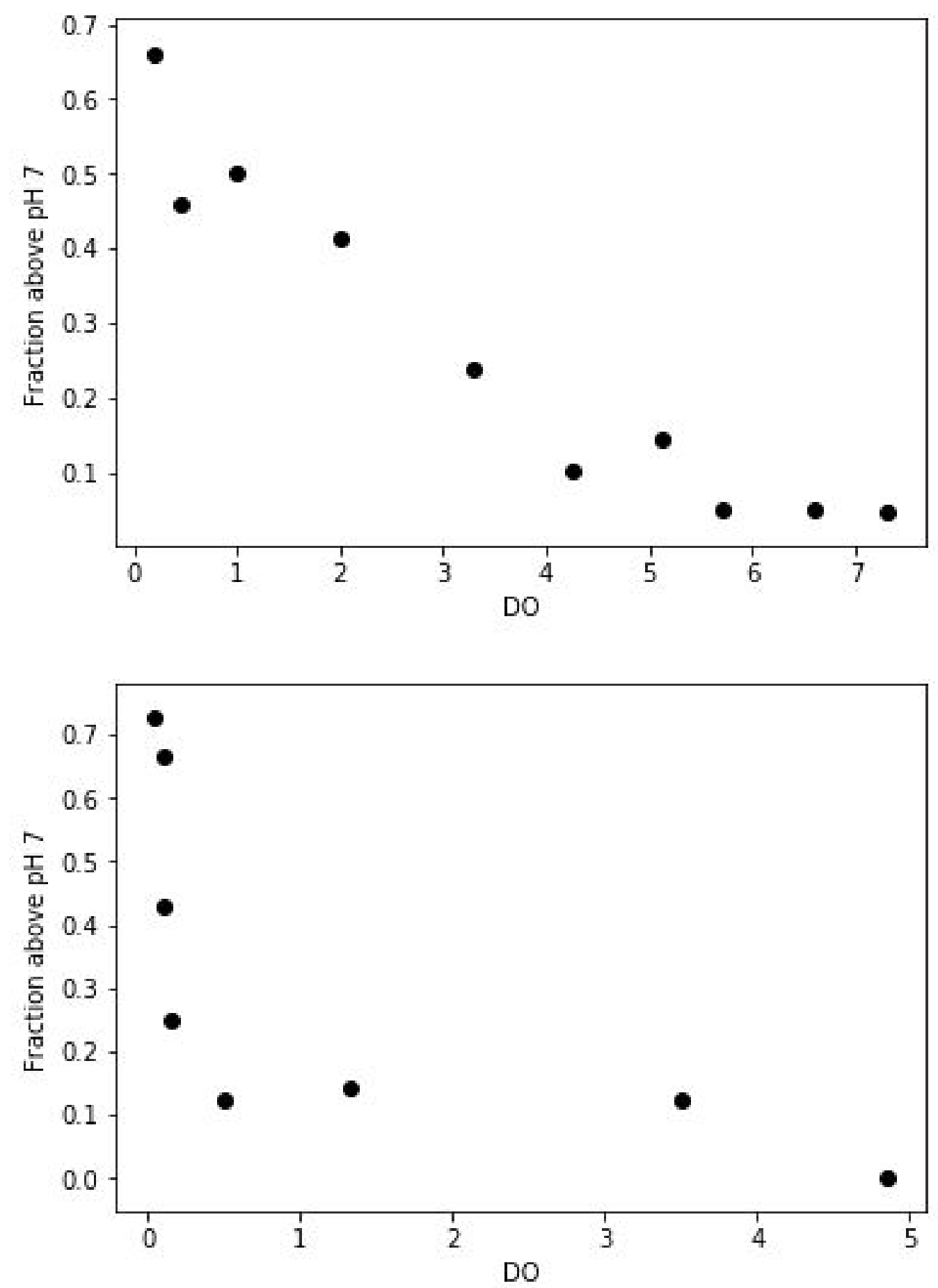

Figure S4. Fraction of samples above neutral pH verses dissolved oxygen concentrations for samples from the Piedmont (top) and Coastal Plain (bottom). 

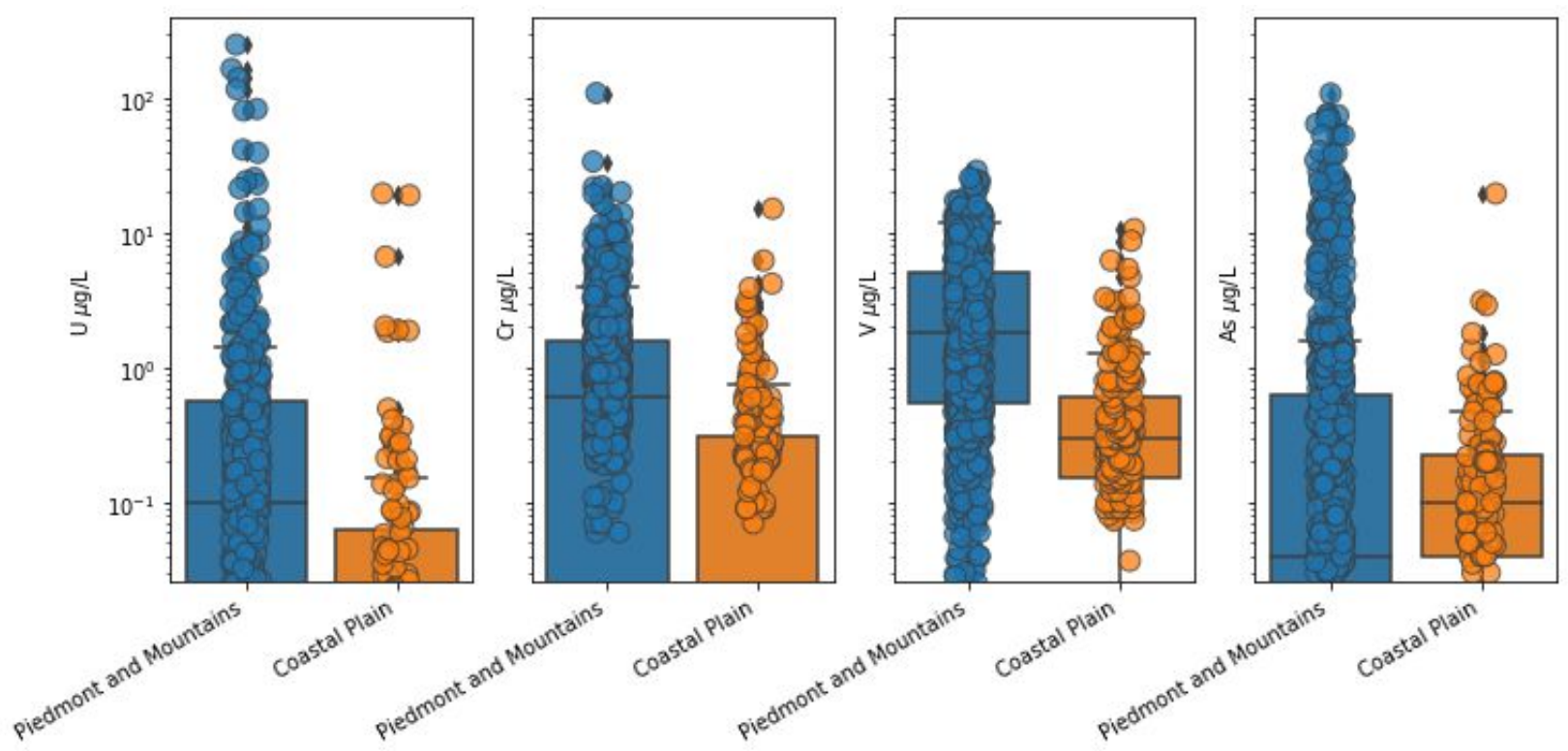

Figure S5. Concentration of RSEs in both the Piedmont and Blue Ridge (PBR) belts and Coastal Plain. With the exception of arsenic, median concentrations of all OFE's were higher in the PBR belts than the Coastal Plain ( $p$-values $<0.01)$. The Coastal Plain has a higher median As concentration than the PRB ( $p$ value $=0.05$ ) 


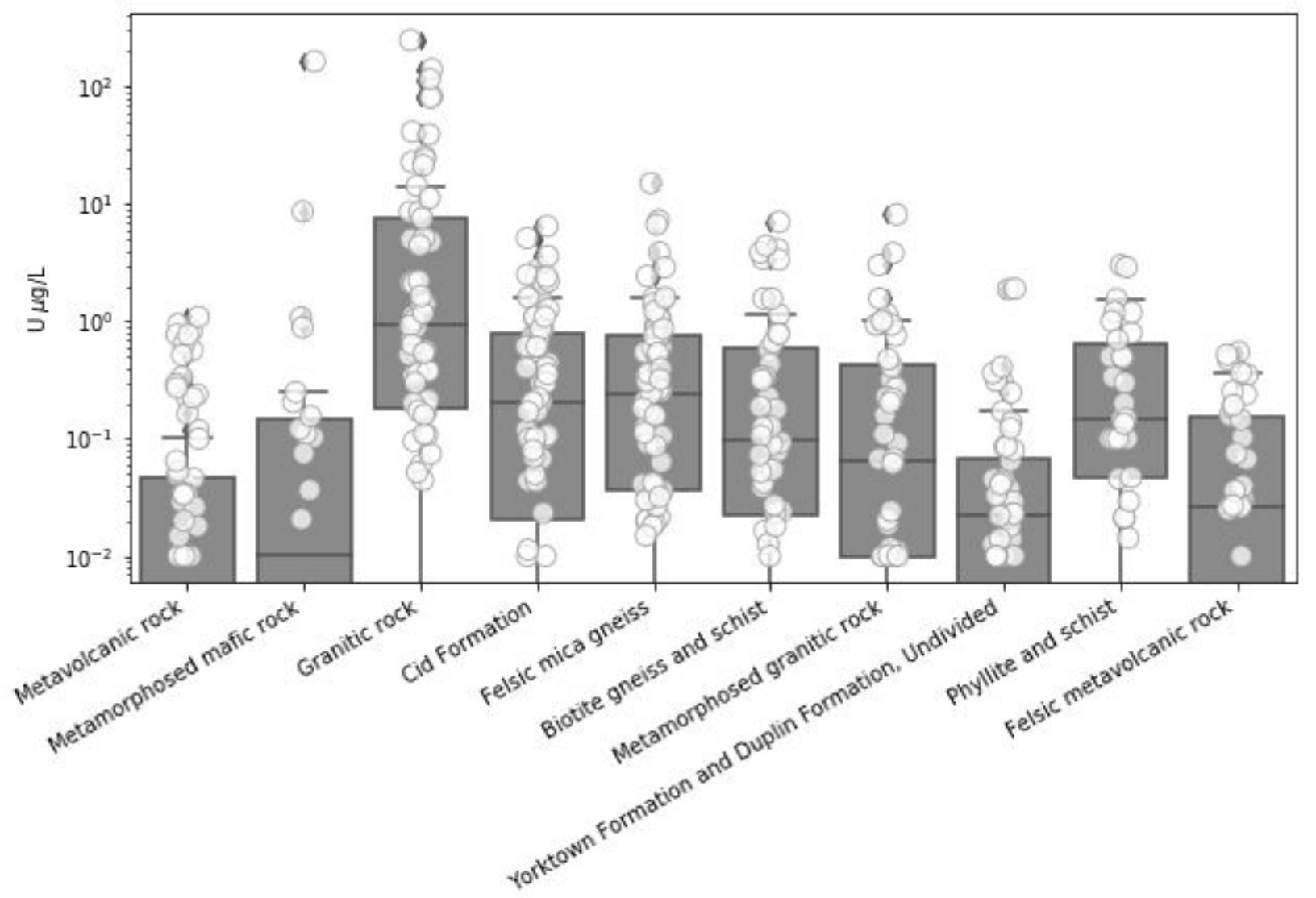

Figure S6. Boxplot of $U$ concentration across formations with at least 20 observations. All of the $U$ concentrations $>100 \mu \mathrm{g} / \mathrm{L}$ are from Granitic formations with the exception of one sample in a metamorphosed mafic rock aquifer which is in an area known to also have granitic masses. 


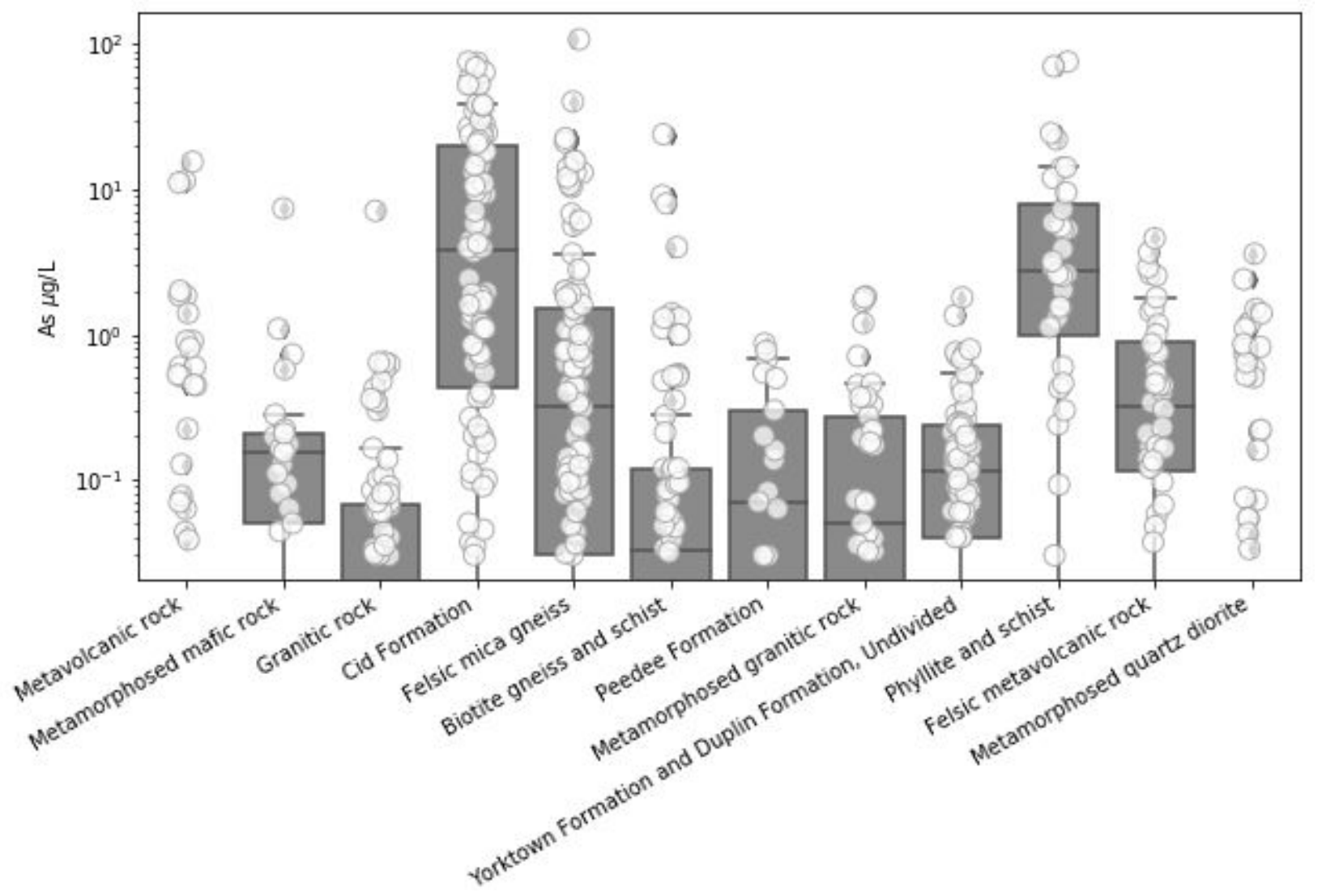

Figure S7. Boxplot of As concentrations across formations with 20 or more observations for As. 


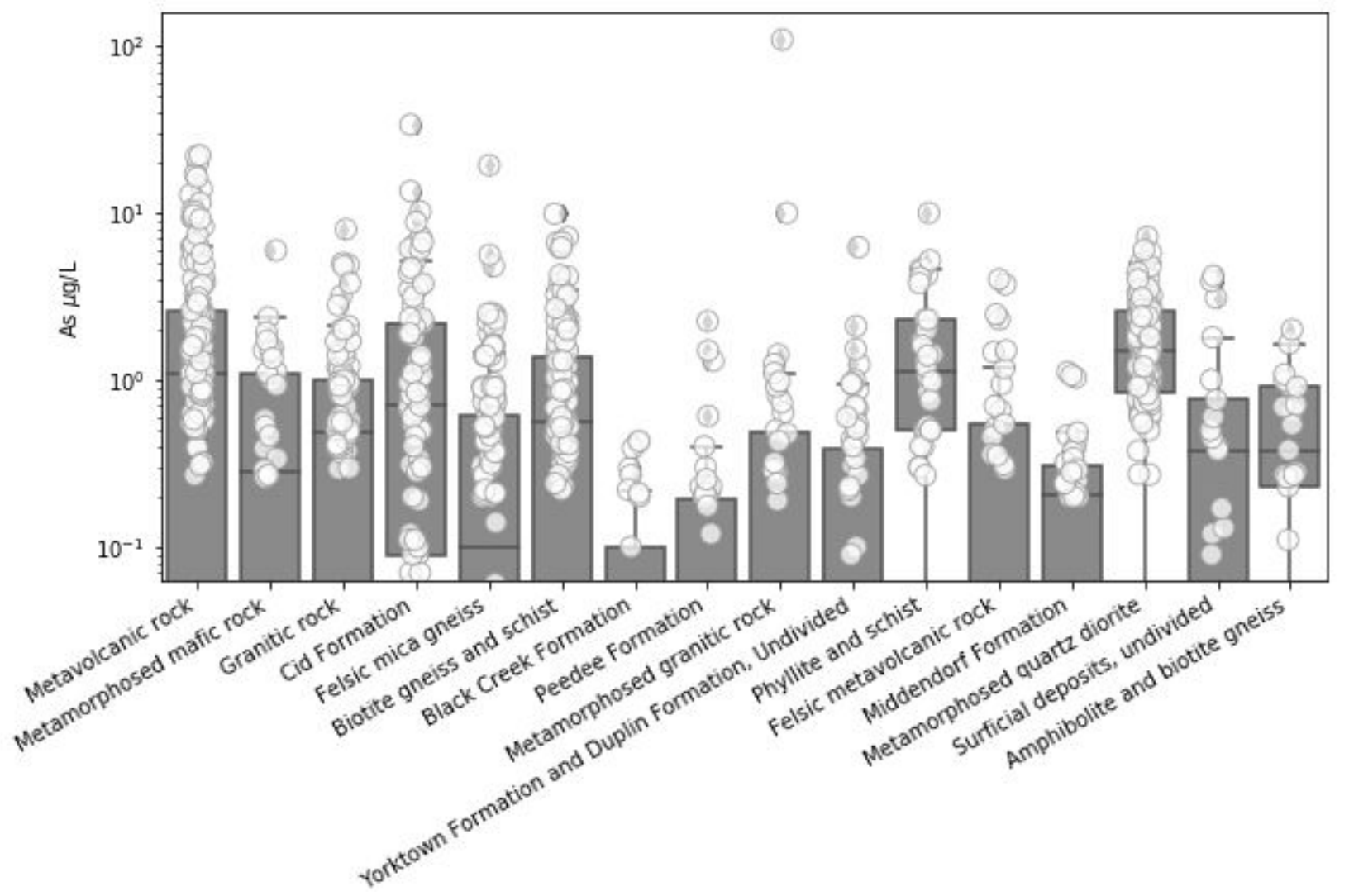

Figure S8. Boxplot of Cr concentrations across formations with 20 or more observations for Cr. 


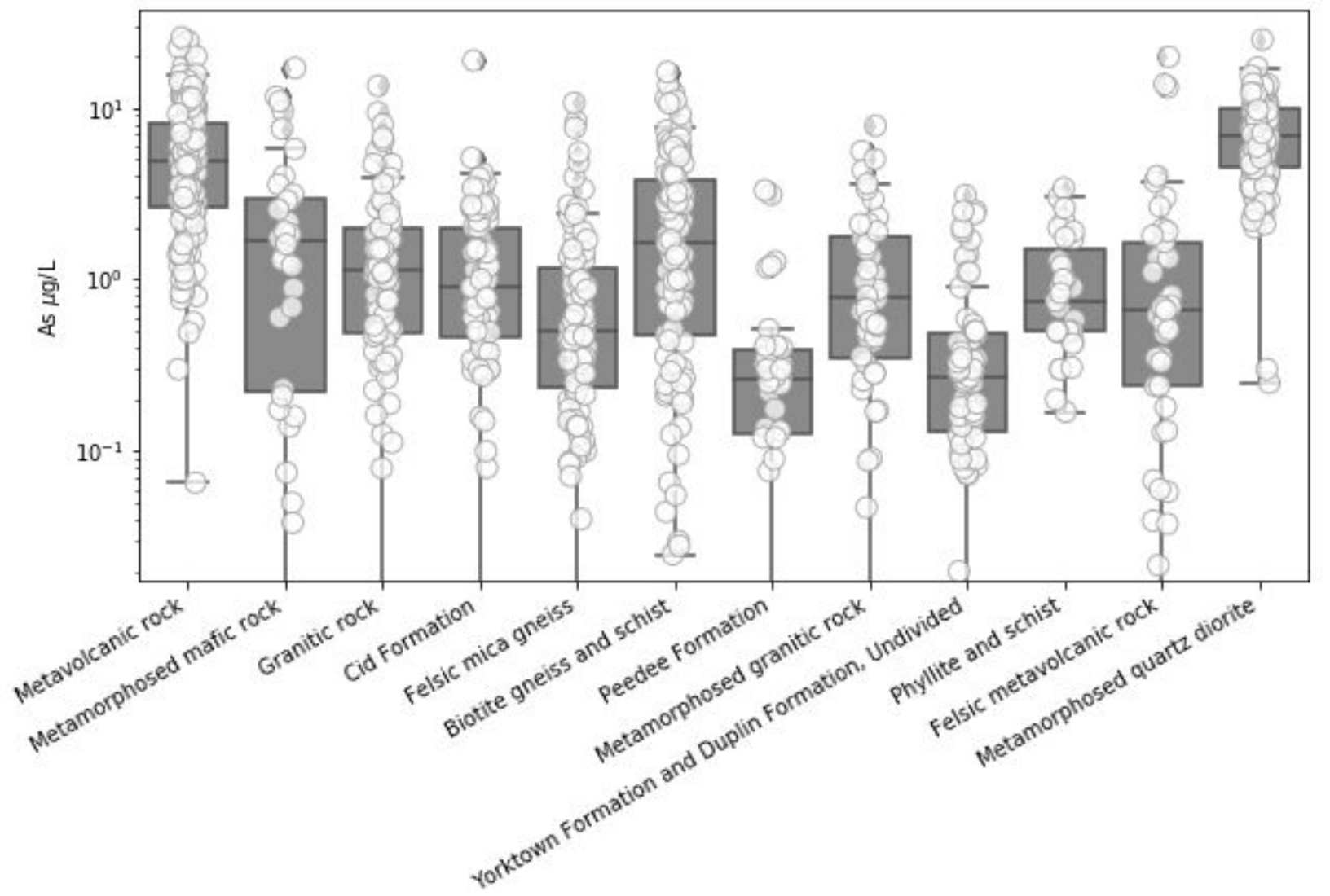

Figure S9. Boxplot of $V$ concentrations across formations with 20 or more observations for $V$. 

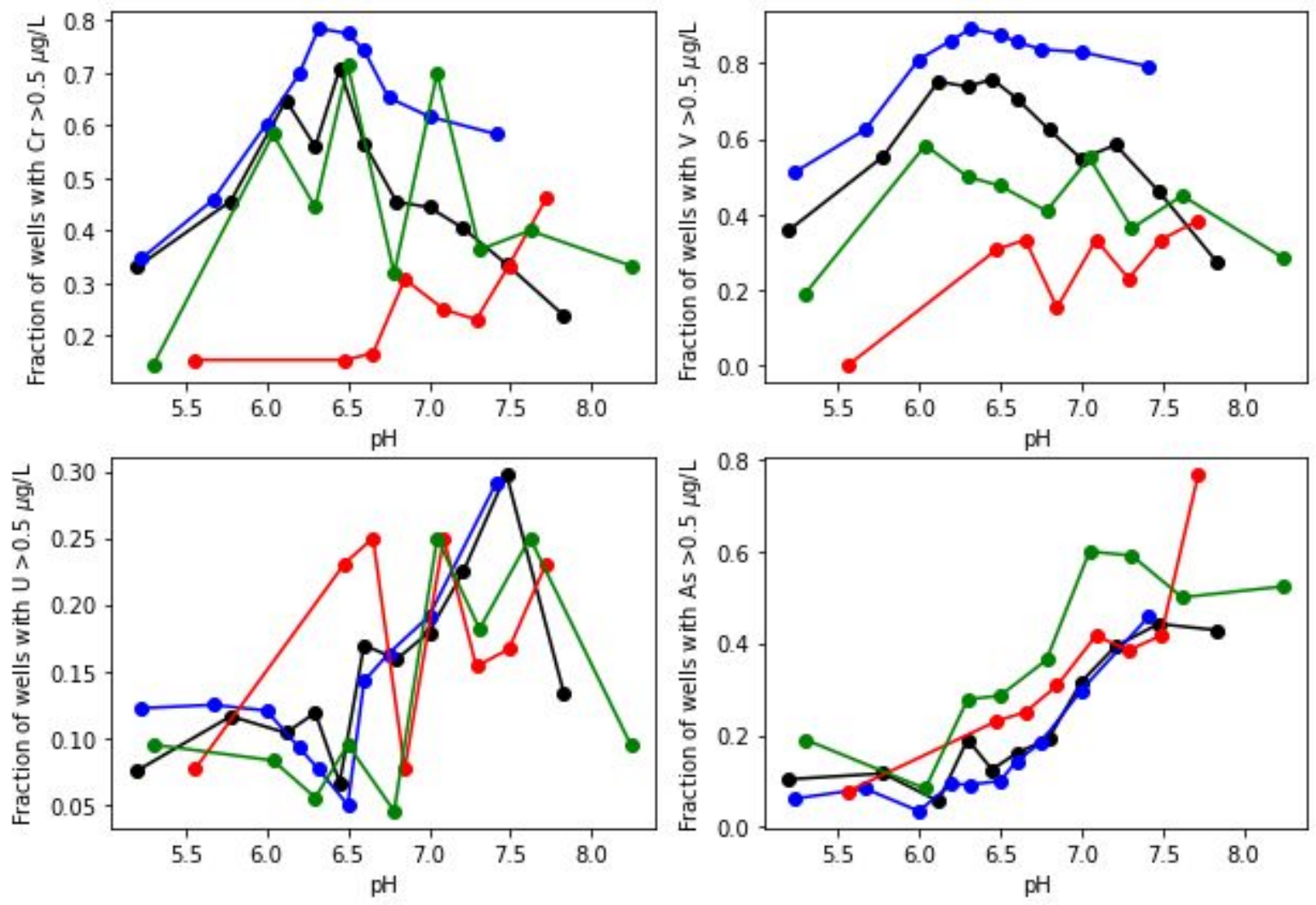

Figure S10. Fraction of wells exceeding $0.5 \mu \mathrm{g} / \mathrm{L}$ vs $\mathrm{pH}$ for each of the studied RSEs. Different colors represent different redox fractions: black is the entire dataset, red is the suboxic fraction, green is the mixed fraction, and blue is the oxic fraction. 

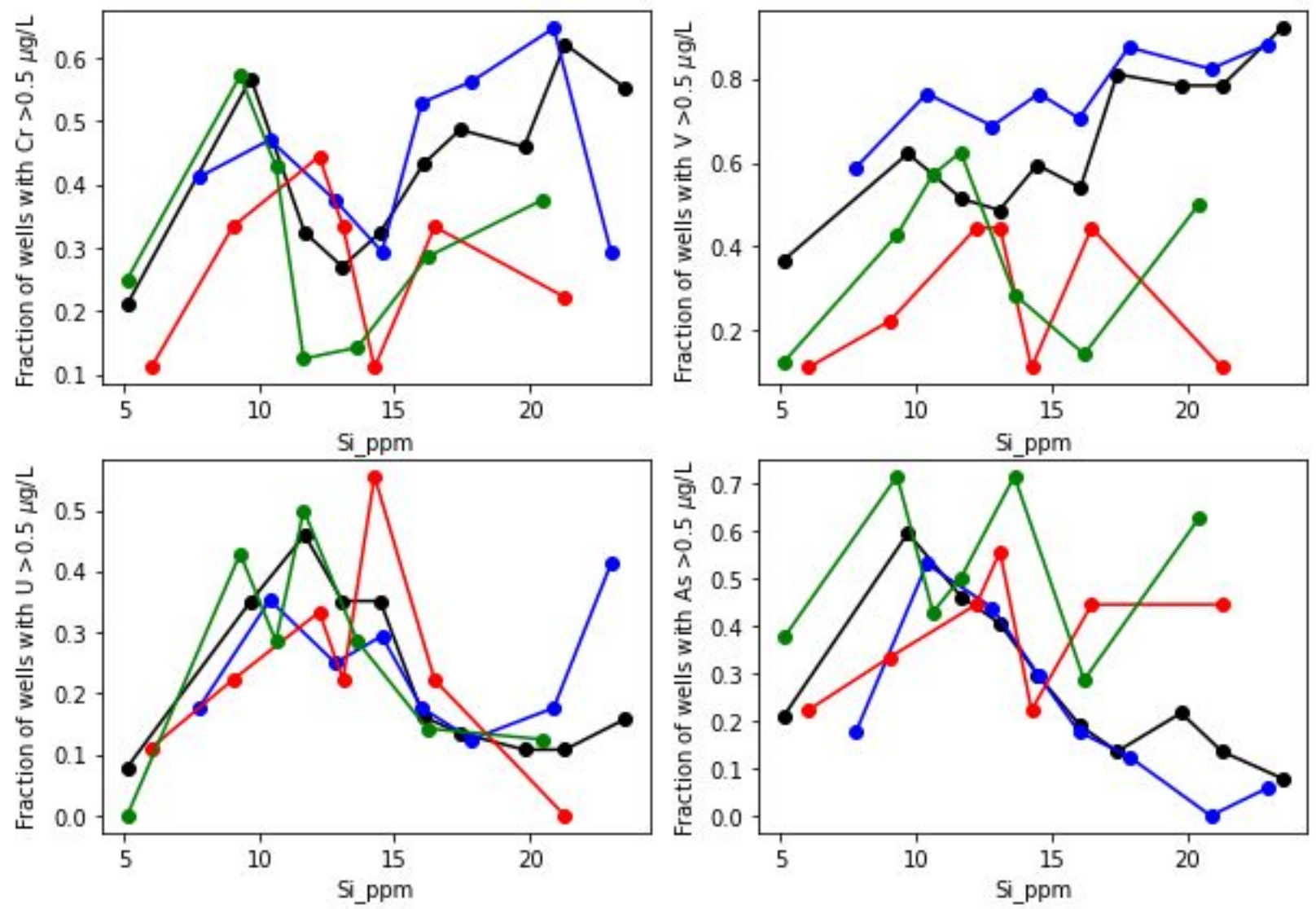

Figure S11. Fraction of wells exceeding $0.5 \mu \mathrm{g} / \mathrm{L}$ vs Si concentration (in $\mathrm{mg} / \mathrm{L}$ ) for each of the studied RSEs. Different colors represent different redox fractions: black is the entire dataset, red is the suboxic fraction, green is the mixed fraction, and blue is the oxic fraction. 

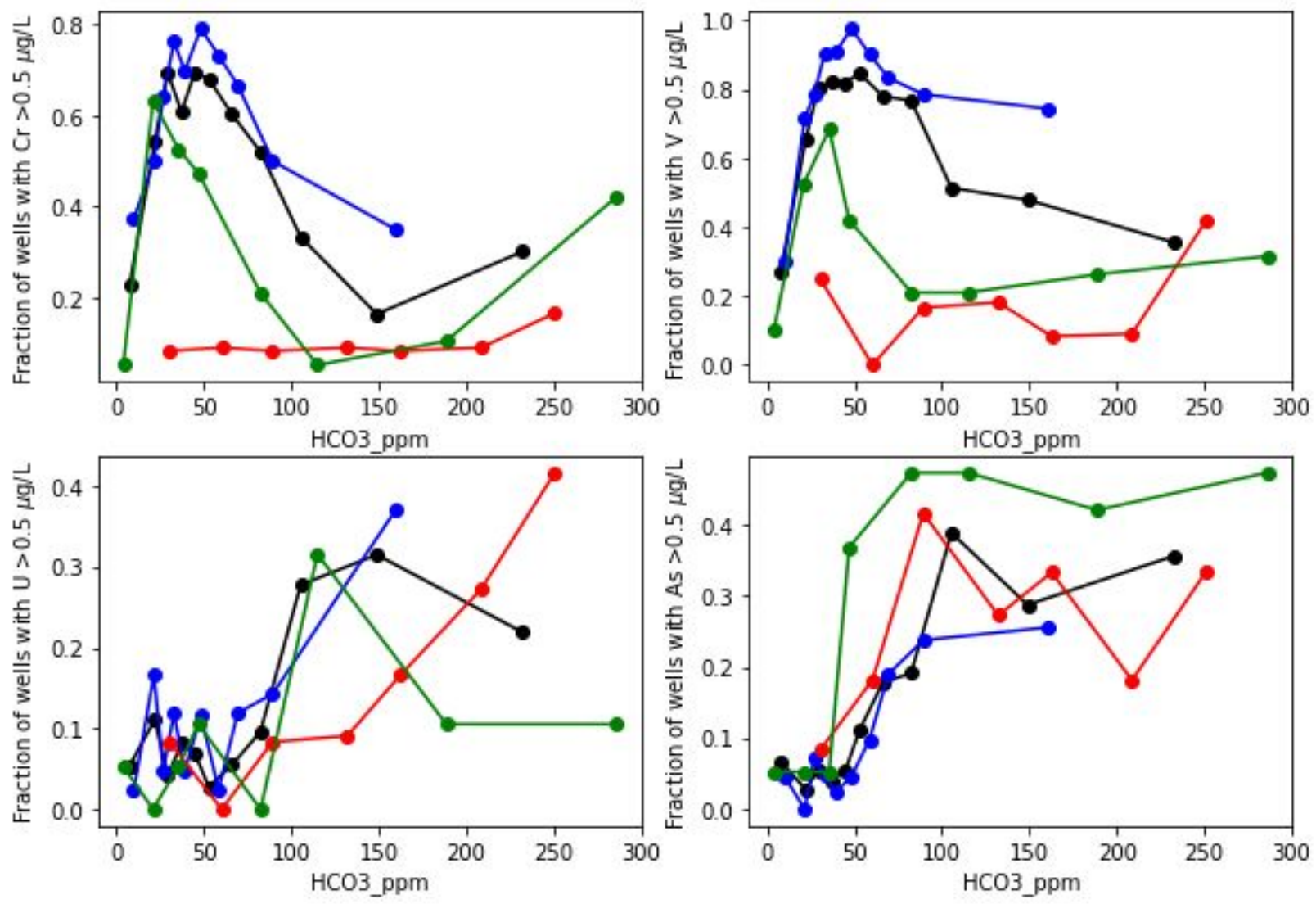

Figure S12. Fraction of wells exceeding $0.5 \mu \mathrm{g} / \mathrm{L} \mathrm{vs} \mathrm{HCO}_{3}{ }^{-}$concentration (in $\mathrm{mg} / \mathrm{L}$ ) for each of the studied RSEs. Different colors represent different redox fractions: black is the entire dataset, red is the suboxic fraction, green is the mixed fraction, and blue is the oxic fraction. 

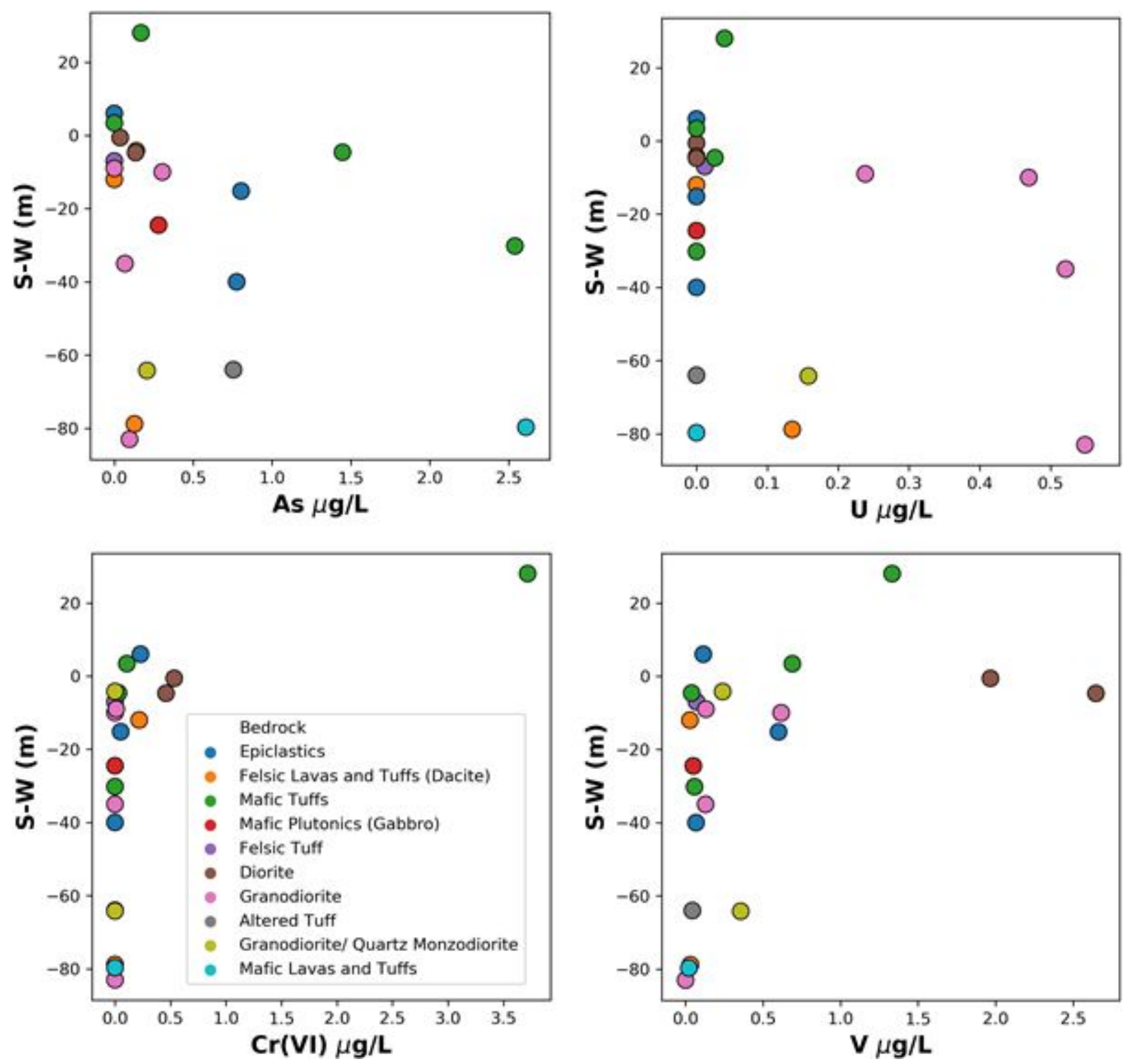

Figure S13. Distance between the depth of the top of the screen and water level $(S-W)$ for monitoring wells in the dataset 

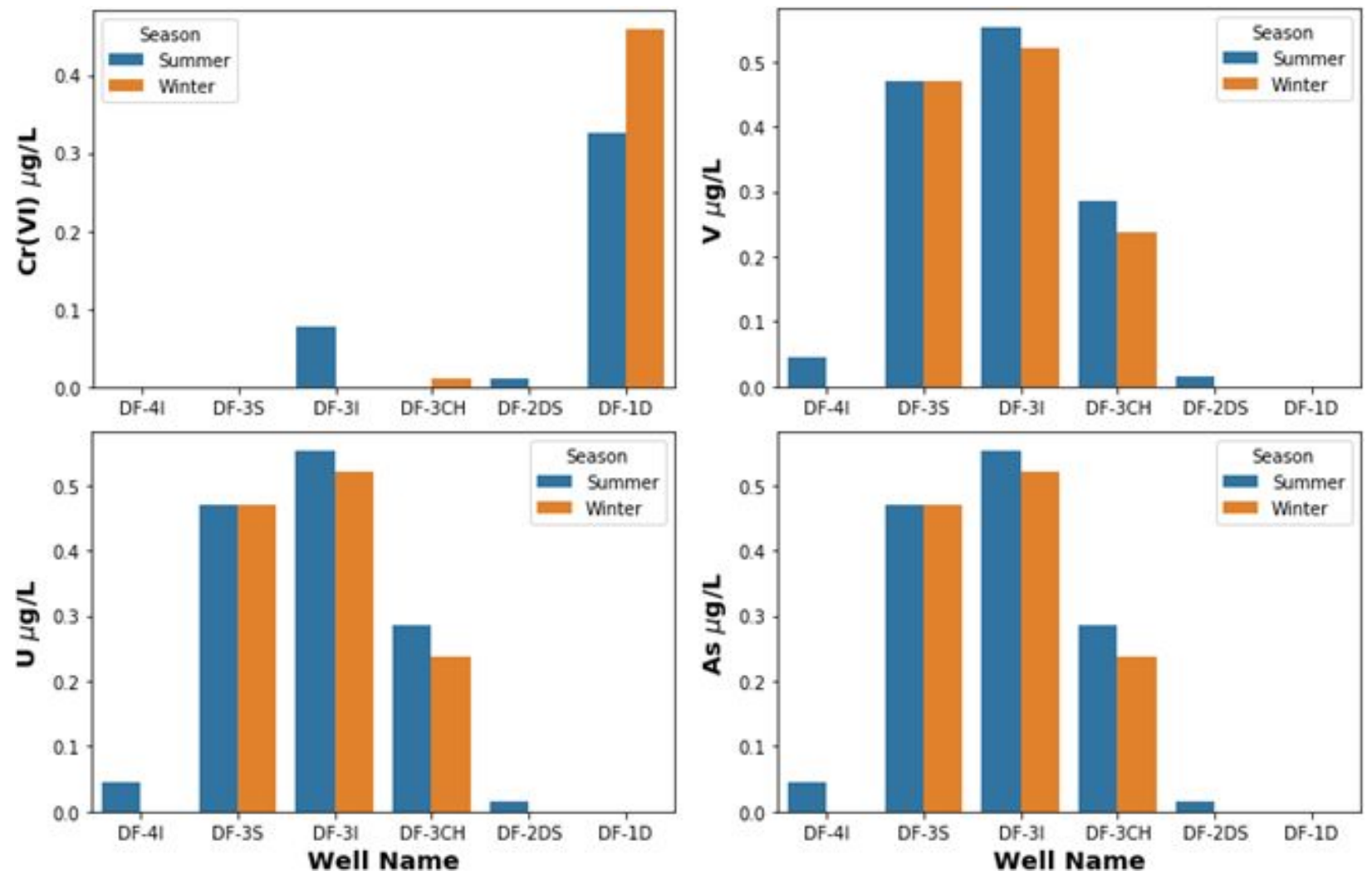

Figure S14. Seasonal variations in oxyanion forming element (OFE) concentrations in Duke Forest monitoring wells. 


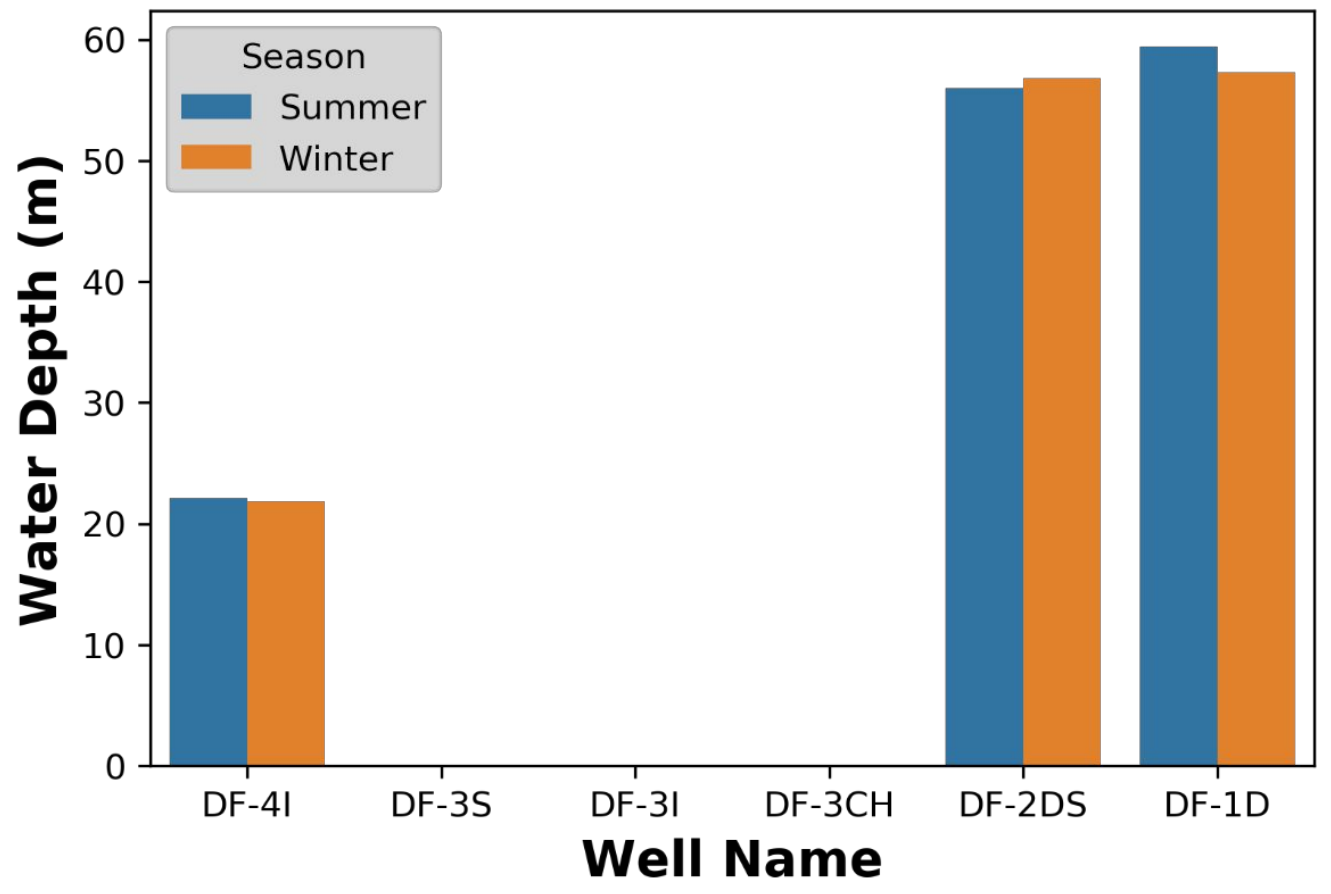

Figure S15. Seasonal variations in water level in Duke Forest monitoring wells. Wells in the DF-3 cluster are artesian. 

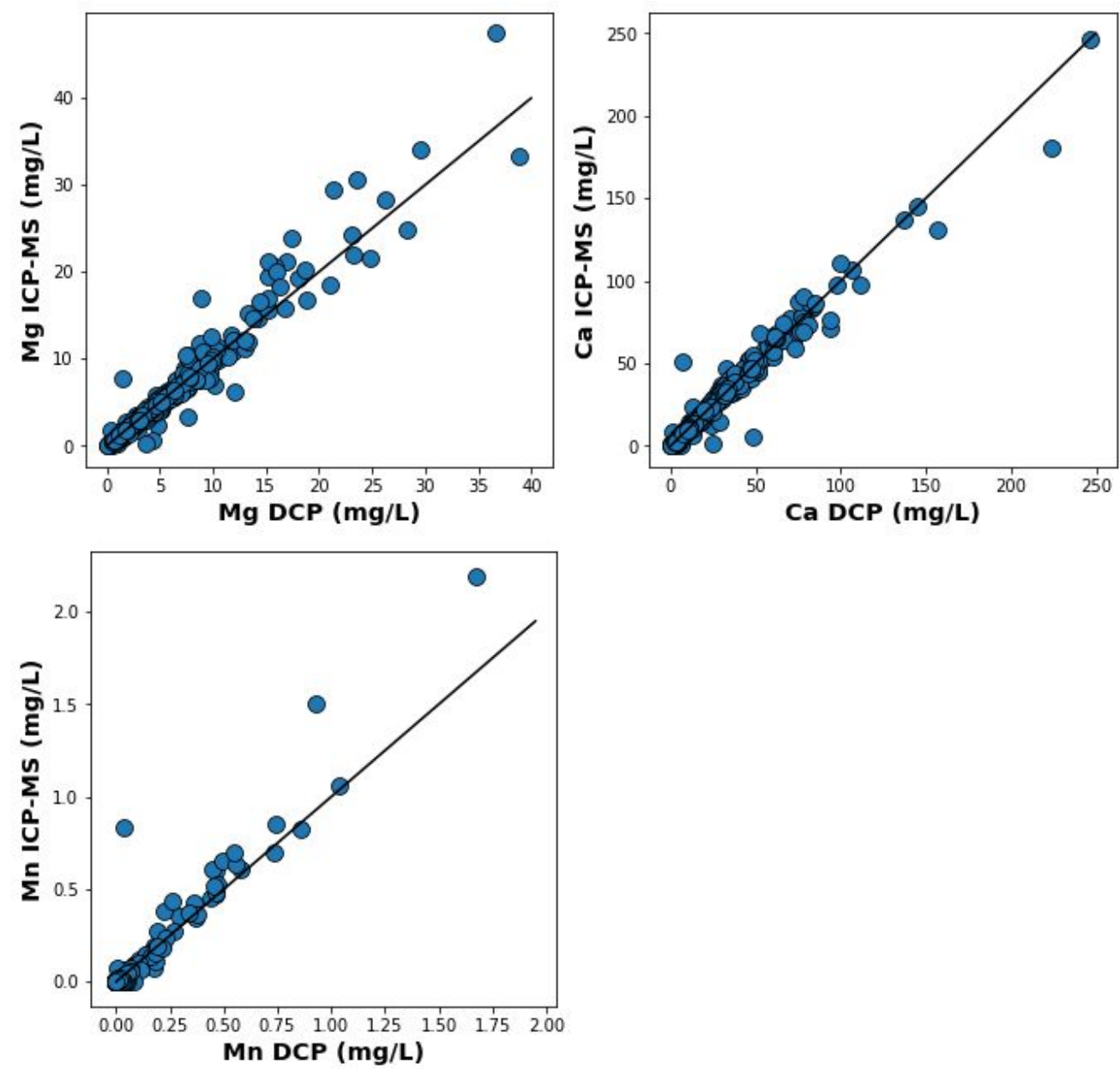

Figure S16. Comparison of measured $\mathrm{Mg}, \mathrm{Ca}$, and Mn values by two different methods, ICP-MS and DCP. Solid lines represent 1:1.

\section{References}

(1) Chapman, M. J.; Cravotta III, C. A.; Szabo, Z.; Lindsey, B. D. Naturally Occurring Contaminants in the Piedmont and Blue Ridge Crystalline-Rock Aquifers and Piedmont Early Mesozoic Basin SiliciclasticRock Aquifers, Eastern United States, 1994-2008; Scientific Investigations Report; United States Geological Survey, 2013.

(2) North Carolina Geological Survey. Geology of North Carolina http://data.nconemap.com/geoportal/catalog/search/resource/details.page?uuid=\%7BA19C03CA6A47-4617-B9BB-F225BAEB0E51\%7D.

(3) Ragland, P. C.; Hatcher Jr, R. D.; Whittington, D. Juxtaposed Mesozoic Diabase Dike Sets from the Carolinas: A Preliminary Assessment. Geology 1983, 11 (7), 394-399. 